Original Research Paper

\title{
Raman Spectroscopy of Protein Crystal Nucleation and Growth
}

\author{
${ }^{1}$ Pechkova Eugenia, ${ }^{2}$ Maksimov, ${ }^{2}$ Georgy, ${ }^{2}$ Parshina Evgenia, ${ }^{2}$ Maksimov Evgenii, \\ ${ }^{2}$ Kutusov Nikolai, ${ }^{2}$ Brazhe Nadezda, ${ }^{2}$ Tarasova Irina, ${ }^{1}$ Stefano Fiordoro and ${ }^{1}$ Nicolini Claudio \\ ${ }^{I}$ Nanoworld Institute Fondazione El.B.A. Nicolini, Biophysics and Nanobiotechnology Laboratories University of \\ Genoa, Genoa, Italy \\ ${ }^{2}$ Biophysics Department, Biological Faculty, Moscow State University, Russian Federation
}

\author{
Article history \\ Received 2014-10-16 \\ Revised 2014-11-05 \\ Accepted 2014-11-07 \\ Corresponding Author: \\ Nicolini Claudio, \\ Nanoworld Institute \\ Fondazione El.B.A. Nicolini, \\ Biophysics and \\ Nanobiotechnology \\ Laboratories University of \\ Genoa, Genoa, Italy \\ E-mail: \\ claudio.nicolini@unige.it
}

\begin{abstract}
Using Raman spectroscopy and the lysozyme as model system, we investigate the differences in protein conformation before and after LangmuirBlodgett nanotemplate-induced crystal nucleation and growth. It was found, that the main difference in lysozyme conformation is associated to the higher amount of S-S bonds in lysozyme of LB crystals, probably in C-end of protein, resulting in the higher stiffness of the lysozyme molecules and LB crystal in a whole. Growth in size of LB crystal over time is also accompanied by the formation of $\mathrm{S}-\mathrm{S}$ bonds. Atomic structure determined by X-ray diffraction correlates to the above pointing to the main differences between LB classical crystals in terms of water molecules environment previously associated to the increased radiation stability of LB crystals.
\end{abstract}

Keywords: Raman Spectroscopy, Thin LB Films, Lysozyme, Crystal Growth

\section{Introduction}

Raman spectroscopy is attractive as a potential diagnostic tool because it requires no extrinsic labeling, is not limited by masking water contributions and is inherently a multiplexing technique. Raman-based measurements of biological samples have already been exploited for the identification of molecular specific markers for disease detection and monitoring (Brazhe et al., 2012). Due to high sensitivity, selectivity and absence of $\mathrm{H}_{2} \mathrm{O}$ interference with measurements Raman spectroscopy is ideal technique to study bond vibrations and conformation of various biomolecules in aqueous solutions. Thus, Raman spectroscopy is widely used to study conformation of isolated molecules and molecules in cells, e.g., erythrocytes (Semenova et al., 2012; Brazhe et al., 2009), cardiomyocytes (Brazhe et al., 2012), bacterial cells (Ashton et al., 2011), viruses (Liu et al., 2005; Dobrov et al., 2014) and other subjects.

We intend to reproduc data published in (Schwartz and Berglund, 1999; 2000) using the modified hanging drop method with the LB nanotemplate of the protein, deposited on the glass cover slide brought in the contact with the protein solution drop. By using this method we obtained both acceleration of the protein nucleation and crystal growth; moreover, the use of the nanotemplate seems to improve both crystal quality and the resistance to the radiation damage (Pechkova et al., 2004; 2009; Belmonte et al., 2012). In several protein systems the nucleation was observed using the template, while classical hanging drop was not successful. Using the lysozyme as the model systems, we expect to understand from the Raman spectra the differences between the mechanism of the crystallization with and without the nanotemplate and estimate the influence of the LB nanotemplate to the protein nucleation and growth. In this study for the first time we present results of RS study of the mechanism of the crystallization with and without the nanotemplate and estimate the influence of the LB nanotemplate to the protein nucleation and growth. Langmuir-Blodgett (LB-this abbreviation should be explained earlier) thin films used as template for protein crystallization develop crystal with improved radiation stability in presence of third generation synchrotron facility (Pechkova and Nicolini, 2001; Nicolini, 1997; Pechkova et al., 2007; 2012; Pechkova and Nicolini, 2010; Pechkova et al., 2005a; 2005b; 2005c). One of the main reason of this success is apparently due to water molecules distribution around protein backbone (Pechkova et al., 2012). 


\section{Materials and Methods}

\section{Crystallization of Lysozyme}

The protein solution used for both the LB and the classical hanging-drop method were of $40 \mathrm{mg} \mathrm{mL}^{-1}$ in 50 $\mathrm{mM}$ Sodium Acetate buffer $\mathrm{pH} 4.5$ at RT. The LB nanotempate method was utilized as described in Pechkova and Nicolini (2001). The protein crystallization polystyrene well (Hampton research) was modified in a such a way to have glass bottom for the Raman spectroscopy measurement. The well high and there for the reservoir volume were decrease in approximately 2 times in respect to commercially available one in order to fit the Raman instrument set-up. Lysozyme thin film was prepared on the water-area interface and compressed to a surface pressure of 25 $\mathrm{mN} / \mathrm{m}$ by means of a Langmuir-Blodgett trough (NTMTD) (Nicolini, 1997). Two protein monolayer was deposited on the siliconized glass cover slide of $20 \mathrm{~mm}$ diameter (Hampton Research) by the LangmuirSchaeffer method. The 4 microliter drop of protein solution and 4 microliter of the precipitant $(0.9 \mathrm{M} \mathrm{NaCl})$ was mixed on the glass slide covered by thin film nanotamplate. The glass slide with the protein template and the drop was sealed on the crystallization well using vacuum grease and equilibrated against $0.6 \mathrm{~mL}$ reservoir filled with precipitant solution $(0.9 \mathrm{M} \mathrm{NaCl})$.

\section{Raman Spectroscopy}

Raman microspectroscopy was used to study conformation of lysozyme in crystalls and to perform qualitative estimation of the change in the amount of S-S bonds. Raman spectra of LB and classical crystals were obtained using complex nanolaboratory NTEGRA Spectra (NT-MDT, Russia) and Nova software (NTMDT, Russia). An inverted optical microscope Olympus IX71 was used for the laser focusing on a sample. NTEGRA spectrometer was operated in a confocal mode during spectrum registration. Raman scattering was detected by CCD-camera cooled with a thermoelectrical cooling system (Peltier element) to $-50^{\circ} \mathrm{C}$. The incident laser light traveled through the glass bottom of crystallization plate and was focused directly on the crystals in hanging drop of protein. Four different crystals of the similar size were measured from each sample, one spectra $240 \mathrm{~s}$ were collected from each crystal with $532 \mathrm{~nm}$ laser, objective $\times 20$ with NA 0.45 , laser power $5.5 \mathrm{~mW}$, grating 600 lines $/ \mathrm{mm}$ with spectral resolution $3.18 \mathrm{~cm}^{-1}$. The measurements take place in 24 , 26,48 and $50 \mathrm{~h}$ after beginning of crystal growth. It must be mentioned that classical crystals grow much slower and at 24 and $26 \mathrm{~h}$ sonly 2 crystals were observed in crystallization plate and only 2 spectra were get.
Fluorescence measurements were performed by time- and wavelength-correlated single photon counting equipment. The setup consists of a photomultiplier system with a Hamamatsu R5900 16channel multi anode Photomultiplier (PML-16, Becker\&Hickl, Berlin, Germany). The polychromator was equipped with 1200 grooves/mm grating resulting in a spectral bandwidth of the PML-16 of $100 \mathrm{~nm}$ (resolution of $6.25 \mathrm{~nm} /$ channel). Excitation was performed with a pulsed $280 \mathrm{~nm}$ (Edinburgh Instruments, UK) laser, $635 \mathrm{~nm}$ BHL700 (Becker\&Hickl, Berlin, Germany) laser and $405 \mathrm{~nm}$ laser diode (IOS, St. Petersburg, Russia) delivering excitation pulses, driven at a repetition rates up to 50 $\mathrm{MHz}$. The fluorescence decay kinetics were approximated by the sum of exponential functions. To compare different kinetic patterns, we calculated the average decay time according to the expression: $\tau_{a v}=\sum_{i}^{n} \tau_{i} a_{i}$, where $\tau_{i}$ is the lifetime of the $i$-th component and $a_{i}$ is the fraction of the amplitude of $i$-th component of the fluorescence decay normalized to $\sum_{i}^{n} a_{i}=1$. All calculations were performed using the Origin 8.0 (OriginLab Corporation, United States) and SPC Image (Becker and Hickl, Germany) software packages.

\section{Data Processing of Raman Spectra}

For the analysis of Raman spectra we employed Raman Cooker (Brazhe, 2014) software developed at Biophysics Department, Moscow State University for the baseline correction. For all spectra we performed baseline subtraction to ensure that the Raman peak intensities were calculated correctly without artificial influence of the baseline drift.

\section{Results and Discussion}

We demonstrated that under the $532 \mathrm{~nm}$ laser excitation Raman spectra of lysozyme crystals have intensive peaks corresponding to indole ring vibrations in Trp, S-S bond vibrations of Cys residues, Phe vibrations, $\mathrm{C}-\mathrm{N}$ and $\mathrm{C}-\mathrm{C}$ stretching, $\mathrm{C}-\mathrm{H}$ bond vibrations and the peptide bond vibration (amide I and amide III) (Figure 1). Assignment of peaks in lysozyme Raman spectra and their sensitivity to the invironment is shown in Table 1. Position of peaks in Raman spectra of lysozyme obtained with $532 \mathrm{~nm}$ laser is the same as we observed previously in lysozyme Raman spectra obtained by $632.8 \mathrm{~nm}$ excitation (Nicolini et al., 2013). The difference of the present study from the previous one (Nicolini et al., 2013) is that here we analyze spectral region 400-1800 $\mathrm{cm}^{-1}$, whereas in (Nicolini et al., 2013) we focused our attention on $500-1100 \mathrm{~cm}^{-1}$ region. We should note, that in spite of the similarity of spectra in region $500-1100 \mathrm{~cm}^{-1}$ excited by 532 and $632.8 \mathrm{~nm}$, there are some differences: The overall spectrum 
intensity at $532 \mathrm{~nm}$ excitation is higher than at $632.8 \mathrm{~nm}$ excitation, especially of Trp peaks 759 and $1008 \mathrm{~cm}^{-1}$; and there is no peak at $634 \mathrm{~cm}^{-1}$ attributed to $\mathrm{S}-\mathrm{H}$ bond vibration in Cys residues under $532 \mathrm{~nm}$ excitation. Such a difference between Raman spectra obtained under excitation with different lasers is well-known for other molecules (Kutuzov et al., 2014; Ul'ianova et al., 2005). The advantage of the present study is that here we can obtain information about secondary lysozyme structure. It is known, that amide I and amide III peaks' position depends on the secondary conformation of protein. Thus, peak positions at 1258 and $1658 \quad\left(<1660 \mathrm{~cm}^{-1}\right)$ corresponds to alpha-helical protein structure. Any shift of these peaks or decrease in their relative intensities is caused be change in the secondary structure and by the appearance of non-structured regions, turns or betasheets. Peak at $508 \mathrm{~cm}^{-1}$ corresponds to vibrations of disulfide bonds between Cys residues and therefore the relative intensity of this peak can be used as a qualitive estimation of the S-S bond amount in lysozyme molecule. There is no information about sensitivity of $1008 \mathrm{~cm}^{-1}$ Trp Raman peak to the invironment or lysozyme conformation. In our experiments we also observed that its intensity was very stable and its absolute values were almost identical in various LB and classical crystals of the same age.

On the basis of these facts we suggest to use 1008 $\mathrm{cm}^{-1}$ peak intensity for the normalization of intensity values of other peaks. We used following ratios of intensities:

- 508 and $1008 \mathrm{~cm}^{-1}\left(\mathrm{I}_{508} / \mathrm{I}_{1008}\right)$ for the qualitative estimation of the relative amount of S-S bonds between Cys residues in lysozyme molecules in classical and LB crystals

- 1258 and $1008 \mathrm{~cm}^{-1}\left(\mathrm{I}_{1258} / \mathrm{I}_{1008}\right)$ and 1658 and 1008 $\left(\mathrm{I}_{1658} / \mathrm{I}_{1008}\right)$ for the qualitative estimation of the relative amount of alpha-helixal structures in lysozyme molecules in classical and LB crystals

- 759 and $1008 \mathrm{~cm}^{-1}\left(\mathrm{I}_{759} / \mathrm{I}_{1008}\right)$ for the evaluation of hydrophobic properties of the micro-environment of indole rings of Trp residues
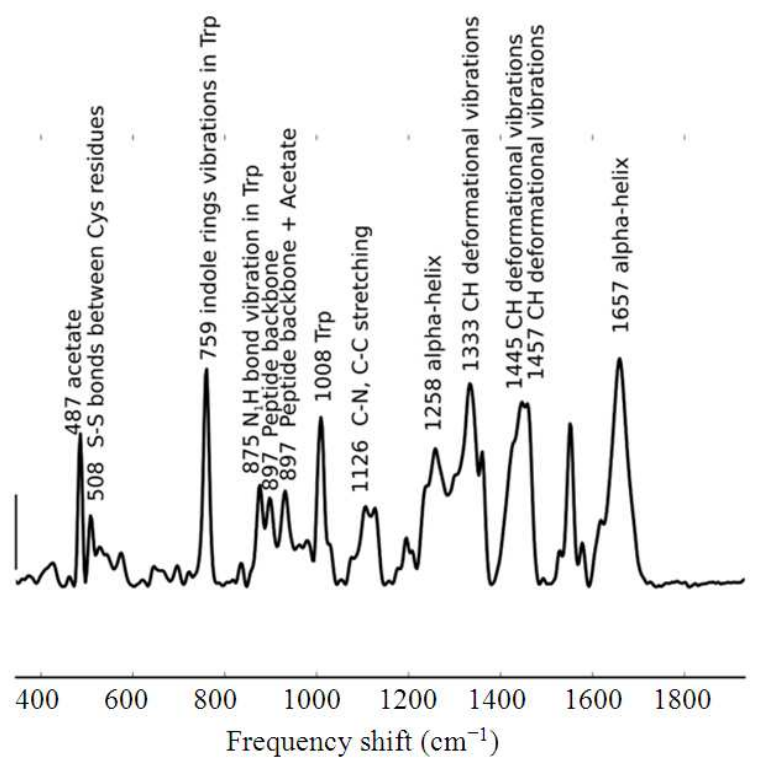

Fig. 1. Raman spectrum of lysozyme crystal at $50 \mathrm{~h}$ of growth. Numbers above peaks show peak maximum position. Vertical scale bar corresponds to 500 a.u. of Raman scattering

Table 1. Assignment of peaks in Raman spectra of lysozyme (Based on (Chandra et al., 2010)). Empty cell in "Sensitivity" column indicates absence of observations of sensitivity of peak intensity or maximum position to the environment or molecule conformational changes.

\begin{tabular}{lll}
\hline Peak position, $\mathrm{cm}^{-1}$ & Assignment & Sensitivity \\
\hline 487 & Bonds of acetate in buffer & \\
508 & S-S bonds between Cys residues & Relative amount of S-S bonds \\
759 & Indole rings in Trp & Hydrophobic properties of the environment \\
875 & $\mathrm{~N}_{1} \mathrm{H}$ bond in Trp & $\begin{array}{l}\text { Frequency shift depends on the strength of H-bond } \\
\text { between Trp and other side-chain AA residue. 865-875 } \\
\end{array}$ \\
& & $\begin{array}{l}\text { in non-bond Trp, 883-in high H-bonding strength Trp } \\
\text { residues }\end{array}$ \\
& &
\end{tabular}

C-N, C-C peptide backbone

$\mathrm{C}-\mathrm{N}, \mathrm{C}-\mathrm{C}$ peptide backbone; acetate in buffer

Ring breathing mode in Trp

C-N, C-C streatching

$\mathrm{C}-\mathrm{N}$ peptide bond (so-called amide III vibration)

$\mathrm{CH}$ deformational vibrations

$\mathrm{CH}$ deformational vibrations

$\mathrm{CH}$ deformational vibrations

C-N peptide bond (so-called amide I vibration)
Secondary structure of protein

Secondary structure of protein: Maximum position at $<1660 \mathrm{~cm}^{-1}$ : Alpha-helix; $>1665 \mathrm{~cm}^{-1}$ random coil or beta-sheets. 
Table 2. Intensity ratios, calculated from Raman spectra of lysozyme LB crystals after various growing time. * $p<0.05$ according to ttest, comparison of LB crystal at $50 \mathrm{~h}$ with LB crystal at 24,26 and $48 \mathrm{~h} ;{ }^{*} \mathrm{p}<0.01$ according to t-test, comparison of LB and classical crystals at $50 \mathrm{~h}$

\begin{tabular}{llllll}
\hline Ratio & Sensitivity & $24 \mathrm{~h}$ & $26 \mathrm{~h}$ & $48 \mathrm{~h}$ & $50 \mathrm{~h}$ \\
\hline $\mathrm{I}_{508} / \mathrm{I}_{1008}$ & Relative amount of S-S bonds & $0.615+0.088$ & $0.527+0.062$ & $0.509+0.061$ & $0.705+0.078^{* \#}$ \\
$\mathrm{I}_{1258} / \mathrm{I}_{1008}$ & Relative amount of alpha-helixes & $1.142+0.099$ & $0.992+0.043$ & $1.068+0.028$ & $1.166+0.084$ \\
$\mathrm{I}_{1658} / \mathrm{I}_{1008}$ & Relative amount of alpha-helixes & $1.99+0.191$ & $1.608+0.043$ & $1.734+0.077$ & $1.744+0.044$ \\
$\mathrm{I}_{759} / \mathrm{I}_{1008}$ & Hydrophobic properties of Trp micro-environment & & $1.35+0.02$ & $1.34+0.01$ & $1.44+0.02^{*}$ \\
\hline
\end{tabular}

Table 3. Intensity ratios, calculated from Raman spectra of lysozyme classical crystals after various growing time. ${ }^{\&} \mathrm{p}<0.05$ according to t-test, comparison of classical crystal at $50 \mathrm{~h}$ with classical crystal at 26 and $48 \mathrm{~h} ;{ }^{\#} \mathrm{p}<0.01$ according to t-test, comparison of LB and classical crystals at $50 \mathrm{~h}$

\begin{tabular}{|c|c|c|c|c|c|}
\hline Ratio & Sensitivity & $24 \mathrm{~h}$ & $26 \mathrm{~h}$ & $48 \mathrm{~h}$ & $50 \mathrm{~h}$ \\
\hline $\mathrm{I}_{508} / \mathrm{I}_{1008}$ & Relative amount of S-S bonds & & $0.579+0.065$ & $0.517+0.07$ & $0.389+0.052^{\text {\&\# }}$ \\
\hline $\mathrm{I}_{1258} / \mathrm{I}_{1008}$ & Relative amount of alpha-helixes & & $0.983+0.078$ & $1.146+0.027$ & $1.084+0.027$ \\
\hline $\mathrm{I}_{1658} / \mathrm{I}_{1008}$ & Relative amount of alpha-helixes & & $1.671+0.071$ & $1.685+0.043$ & $1.742+0.047$ \\
\hline $\mathrm{I}_{759} / \mathrm{I}_{1008}$ & Hydrophobic properties of Trp micro-environment & & $1.37+0.09$ & $1.37+0.04$ & $1.42+0.04^{\&}$ \\
\hline
\end{tabular}

We showed that (Table 2 and 3):

- Within the growing time LB crystals show tendency to the increase in the relative amount of S-S bonds between Cys residues. The deference is significant at $50 \mathrm{~h}$ after starting the growing. There is no change in the relative amount of alpha-helixes with the growing time

- Within the growing time classical crystals do not change amount of alpha-helixes, but demonstrate significant decrease in the amount of disulfide bonds between Cys residues at $50 \mathrm{~h}$ in the comparison with 26 and $48 \mathrm{~h}$

- We did not observe any significant difference in vibrations of Trp residues sensitive to hydrophobic properties of the Trp indole ring surrounding. This means that hydrophobic properties of the micro-environment of Trp indole rings are similar in LB and classical crystal at each time point after nucleation. At the same time we observed significant increase in hydrophobic properties in both crystals at $50 \mathrm{~h}$ in the comparison to crystals at 26 and $48 \mathrm{~h}$

- Comparison between classic crystals and LB crystals show that in $50 \mathrm{~h}$ after starting the procedures of LB and classic crystal growing LB crystals have higher amount of $\mathrm{S}-\mathrm{S}$ bonds. We showed that with the crystal growth after $50 \mathrm{~h}$ there is a tendency to the decrease in the relative amount of alpha-helixes in lysozyme molecules in LB crystals than comparing to classical crystals

We should also note that after first $24 \mathrm{~h}$ classical crystals were so small that it was impossible to record Raman spectra, where as LB crystals were well-seen after $24 \mathrm{~h}$. Importantly, comparison of LB or classical crystals of different sizes at the same time point after the nucleation does not reveal any spectral changes. This demonstrate that the amount of S-S bonds or alphahelixes does not depend on the crystal size and that observed spectral differences between LB and classical crystalls at the same growing time are due to the internal properties and not due to the possible size difference.

The data about change in the amount of S-S bonds are in the agreement with our previous results (Nicolini et al., 2013). To relate spectral changes with the conforamtional difference of lisozyme molecules in LB and classical crystals we used following information: There are four possible disulfide bonds in lisozyme. Two of them S6/S127 and S30/S115 locate closely to C-end of lysozyme that is more flexible and susceptible to the environment than other lisozyme regions including $\mathrm{N}$-terminal (Chandra et al., 2005). Formation of S-S bonds in C-terminal that affects the conformation of the C-terminal and, possibly, the whole lysozyme. We can speculate that due to the higher amount of disulfide bonds C-terminal is more rigid in LB cristals than in classic crystals that can produce more rigid and stable crystals.

Depolarization ratio reflects the anisotropy in Raman scattering and depends on the relative orientations of protein secondary structure elements (alpha spirals and beta-sheets) as well as on the orientation of major crystal axis relative to the coverslip (the one on which crystals grow). Comparison of depolarization ratio in Control (C) and Modified (M) crystalline objects. Modified (M) crystals were grown on special substrate. There is no significant difference in depolarization ratio between two objects (T-test $p$ value $=0.22$ ). The Depolarization Ratio (DR) in the case of lysozyme cannot provide detailed information upon orientation of various elements of secondary structure. In the current experiments we've measured the averaged value of DR. However, from obtained data it seems likely that (C) and (M) samples doesn't differ in relative orientation 
between various secondary structure elements. In other words the conformation of protein is conserved. This result is consistent with the data about absence of the significant change in the relative amount of alpha-helixes in LB and classical crystals.

The results is showing difference in fluorescence decay lifetime indicating structural or environmental differences of tryptophan residuals

\section{Conclusion}

Raman technology provided us a powerful technology to probe the structural alteration apparent in proteins during their crystallization process induced by LB nanotemplate, which parallel the water molecules redistribution in protein crystal in presence of LB protein nanotemplate (Pechkova et al., 2007; 2012).

In particular, in this manuscript we focused on application of traditional and polarized Raman spectroscopies and fluorescent spectroscopy to the study of lysozyme molecule conformation in crystals under growth process with classical method or with LB nanotemplate method. Previously we demonstrated that how the amount of the water molecules and their arrangement affected the crystallization process and we also performed pilot Raman study to compare lysosyme conformation in different LB and classical crystals. At present paper we report detailed study of lysozyme properties in LB and classical crystals within the growing time. We suggest that one of the main differences in lysozyme conformation in LB and classical crystals is caused by higher amount of $\mathrm{S}-\mathrm{S}$ bonds in lysozyme of LB crystals, probably in C-end of protein. This can result in the higher stiffness of lysozyme molecules and LB crystal in a whole.

\section{Acknowledgement}

This project was supported by MIUR (Ministero dell'Istruzione, Università e Ricerca) to Fondazione Elba Nicolini with annual grants for "Funzionamento" and to Professor Claudio Nicolini at the University of Genova for FIRB Italnanoitalnet RBPR05JH2P_04.. We are gratefull to Carpentier Philippe for his assistance in collecting the Raman data at the Cryobench of ID23 at ESRF and to Alexey Brazhe for the help with Raman data analysis. diffusion hanging drop.

\section{Author's Contributions}

All authors equally contributed in this work.

\section{Ethics}

This article is original and contains unpublished materials. The corresponding author confirms that all of the other authors have read and approved the manuscript and no ethical issues involved.

\section{References}

Ashton, L., K. Lau, C.L. Winder and R. Goodacre, 2011. Raman spectroscopy: Lighting up the future of microbial identification. Future Microbiol., 6: 991997. DOI: $10.2217 / \mathrm{fmb} .11 .89$

Belmonte, L., E. Pechkova, S. Tripathi, D. Scudieri and C. Nicolini, 2012. Langmuir-blodgett nanotemplate and radiation resistance in protein crystals: State of the art. Critical Rev. Eukaryotic Gene Exp., 22: 219232.

DOI: 10.1615/CritRevEukarGeneExpr.v22.i3.50

Chandra, S., G. Gallardo, R. Fernández-Chacón, O.M. Schlüter and T.C. Südhof, 2005. Alpha-synuclein cooperates with CSPalpha in preventing neurodegeneration. Cell, 4: 383-396. PMID: 16269331

Brazhe, N.A., M. Treiman, A.R. Brazhe, N.L. Find and G.V. Maksimov et al., 2012. Mapping of redox state of mitochondrial cytochromes in live cardiomyocytes using Raman microspectroscopy. PloS One, 7: e41990-e41990. DOI: 10.1371/journal.pone.0041990

Brazhe, N.A., S. Abdali, A.R. Brazhe, O.G. Luneva and N.Y. Bryzgalova et al., 2009. New Insight into Erythrocyte through In Vivo Surface-Enhanced Raman Spectroscopy. Biophys. J., 97: 3206-3214. DOI: $10.1016 /$ j.bpj.2009.09.029

Chandra, G., K.S. Ghosh, S. Dasgupta and A. Roy, 2010. Evidence of conformational changes in adsorbed lysozyme molecule on silver colloids. Int. J. Biol. Macromolecules, 47: 361-365. DOI: 10.1016/j.ijbiomac.2010.05.020

Dobrov, E.N., N.A. Nikitin, E.A. Trifonova, E.Y. Parshina and V.V. Makarov et al., 2014. $\beta$-structure of the coat protein subunits in spherical particles generated by tobacco mosaic virus thermal denaturation. J. Biomolecular Structure Dynam., 32: 701-708. DOI: 10.1080/07391102.2013.788983

Kutuzov, N.P., A.R. Brazhe, G.V. Maksimov, O.E. Dracheva and V.L. Lyaskovskiy et al., 2014. Orientational ordering of carotenoids in myelin membranes resolved by polarized Raman microspectroscopy. Biophys. J., 107: 891-900. DOI: 10.1016/j.bpj.2014.07.002

Liu, W.L., K. Alim, A.A. Balandin, D.M. Mathews and J.A. Dodds, 2005. Assembly and characterization of hybrid virus-inorganic nanotubes. Applied Phys. Lett., 86: 253108-253108. DOI: 10.1063/1.1952587

Nicolini, C., 1997. Protein-monolayer engineering: Principles and application to biocatalysis. Trends Biotechnol., 5: 395-401. DOI: 10.1016/S01677799(97)01084-6 
Nicolini, C., L. Belmonte, G. Maksimov, N. Brazhe and E. Pechkova, 2013. In situ monitoring by raman spectroscopy of lysozyme conformation during "nanotemplate" induced crystallization. J. Microb. Biochem. Technol., 6: 009-016. DOI:10.4172/19485948.1000114

Pechkova, E. and C. Nicolini, 2001. Accelerated protein crystal growth by protein thin film template. J. Crystal Growth, 231: 599-602. DOI: 10.1016/S0022-0248(01)01450-6

Pechkova, E. and C. Nicolini, 2010. D

omain organization and properties of LB lysozyme crystals down to submicron size. Anticancer Res., 30: 2745-2748. PMID: 20683008

Pechkova, E., G. Tropiano, C. Riekel and C. Nicolini, 2004. Radiation stability of protein crystals grown by nanostructured templates: Synchrotron microfocus analysis. Spectrochimica Acta, B59: 1687-1693. DOI: 10.1016/j.sab.2004.07.020

Pechkova, E., S. Fiordoro, D. Fontani and C. Nicolini, 2005a. Investigating crystal-growth mechanisms with and without LB template: Protein transfer from LB to crystal. Acta Crystallographica, D61: 809812. DOI: $10.1107 /$ S0907444905006566

Pechkova, E., V. Sivozhelezov, G. Tropiano, S. Fiordoro and C. Nicolini, 2005b. Comparison of lysozyme structures derived from thin-film-based and classical crystals. Acta Crystallographica, D61: 803-808. DOI: $10.1107 /$ S0907444905006578

Pechkova, E., F. Vasile, R. Spera and C. Nicolini, 2005c. Protein nanocrystallography: Growth mechanism and atomic structure of crystals induced by nanotemplates. J. Synchrotron Radiat., 12: 772-778. DOI: $10.1107 /$ S0909049505011647
Pechkova, E., V. Sivozhelezov and C. Nicolini, 2007. Protein thermal stability: The role of protein structure and aqueous environment. Arch. Biochem. Biophys., 466: 40-48. DOI: 10.1016/j.abb.2007.07.016

Pechkova, E., S. Tripathi, R.B.G. Ravelli, S. McSweeney and C. Nicolini, 2009. Radiation stability of proteinase $\mathrm{K}$ crystals grown by LB nanotemplate method. J. Strucutarl Biol., 168: 409-418. DOI: 10.1016/j.jsb.2009.08.005

Pechkova, E., V. Sivozhelezov, L. Belmonte and C. Nicolini, 2012. Unique water distribution of Langmuir-Blodgett versus classical crystals. J. Structural Biol., 180: 57-64. DOI: 10.1016/j.jsb.2012.05.021

Schwartz, A.M. and K.A. Berglund, 1999. The use of Raman spectroscopy for in situ monitoring of lysozyme concentration during crystallization in a hanging drop. J. Crystal Growth, 203: 599-603. DOI: $10.1016 / \mathrm{S} 0022-0248(99) 0011$

Schwartz, A.M. and K.A. Berglund, 2000. In situ monitoring and control of lysozyme concentration during crystallization in a hanging drop. J. Crystal Growth, 210: 753-760. DOI: 10.1016/S00220248(99)00423-6

Semenova, A.A., E.A. Goodilin, N.A. Brazhe, V.K. Ivanov and A.E. Baranchikov et al., 2012. Planar SERS nanostructures with stochastic silver ring morphology for biosensor chips. J. Mater. Chem. 22: 24530-24544. DOI: 10.1039/C2JM34686A

Ul'ianova, N.A., G.V. Maksimov, A.A. Churin and A.B. Rubin, 2005. Effect of nitric oxide on viscosity of nerve cell membranes. Biofizika, 50: 289-296. PMID: 15856987

Brazhe, A., 2014. Pyraman: Working with Raman spectra in Python. Atlassian in San Francisco. 